\section{Avaliação do nível de conhecimento e de atitudes preventivas da população sobre a leishmaniose visceral em Belo Horizonte, Minas Gerais, Brasil}

\author{
Assessment of knowledge and preventive attitudes \\ concerning visceral leishmaniasis in Belo Horizonte, \\ Minas Gerais State, Brazil
}

\author{
${ }^{1}$ Escola de Veterinária, \\ Universidade Federal de \\ Minas Gerais, Belo Horizonte, \\ Brasil. \\ ${ }^{2}$ Gerência de Controle de \\ Zoonoses, Prefeitura de \\ Belo Horizonte, \\ Belo Horizonte, Brasil. \\ Correspondência \\ B. K. A. Borges \\ Departamento de Medicina \\ Veterinária Preventiva, \\ Escola de Veterinária, \\ Universidade Federal de \\ Minas Gerais. \\ Rua Oito 115, Montes Claros, \\ MG 39400-000, Brasil. \\ biakborges@hotmail.com \\ barbarakellen@ig.com.br
}

\begin{abstract}
The main objective of this study was to evaluate knowledge concerning visceral leishmaniasis and attitudes used to prevent the disease in Belo Horizonte, Minas Gerais State, Brazil, in 2006. A casecontrol study was conducted, with home visits and a questionnaire. The odds ratio was calculated, comparing 82 cases of human visceral leishmaniasis in 2004 and 164 controls (neighbors of cases). The disease was more frequent in children (OR = 109.77). Visceral leishmaniasis was 2.57 times more likely in males than in females. Overall schooling level was low (68.3\% of subjects had not completed secondary school). Half of the cases did not know what visceral leishmaniasis was, and only $1.2 \%$ could identify the vector. Having basic knowledge of visceral leishmaniasis reduced the odds of acquiring the disease by 2.24 times. Keeping the house clean and taking pet dogs to the veterinarian reduced the risk by 1.94 times. In Belo Horizonte, the population's level of knowledge on visceral leishmaniasis is superficial, and preventive attitudes are inaccurate.
\end{abstract}

Visceral Leishmaniasis; Risk Factors; Knowledge; Disease Prevention
Bárbara Kellen Antunes Borges 1 José Ailton da Silva ${ }^{1}$

João Paulo Amaral Haddad 1

Élvio Carlos Moreira 1

Danielle Ferreira de Magalhães 1

Letícia Mendonça Lopes Ribeiro ${ }^{1}$

Vanessa de Oliveira Pires Fiúza 2

\section{Introdução}

A leishmaniose visceral é uma zoonose de grande relevância na saúde coletiva devido à sua heterogeneidade epidemiológica, alta letalidade em pacientes não tratados e soroprevalência 1 . O controle da doença tem sido realizado pela adoção de três medidas básicas: o tratamento dos casos humanos, a eutanásia de cães soropositivos e a redução da população de vetores, através da aplicação de inseticida nos domicílios situados em área endêmica 2,3.

Mas, apesar dos esforços, a leishmaniose visceral tem aumentado significativamente sua importância no contexto epidemiológico em decorrência do processo de urbanização e das alterações no ambiente natural 4. A forte relação apresentada pela ocorrência da leishmaniose visceral e os perfis cultural, nutricional e sócioeconômico da população atingida, remetem a questão do controle para além das barreiras pertencentes ao contexto ambiental em que a doença está inserida 5 .

Em Belo Horizonte, Minas Gerais, Brasil, a leishmaniose visceral tem-se expandido mesmo com uso de grande recurso financeiro e humano no seu controle. Durante seu recente histórico na cidade (iniciado em 1992) a doença alcançou altos coeficientes de morbidade e letalidade nas populações atingidas $4,6,7,8$.

Segundo Dias 9, o êxito das estratégias contra as doenças endêmicas depende basicamen- 
te da disponibilidade de recursos econômicos e, necessariamente, do conhecimento das competências e atitudes da população diante dos problemas mórbidos, relevantes para a aceitação e participação efetivas nas ações profiláticas.

Luz et al. 10, citam a educação como controle cultural para a leishmaniose visceral, por tornar participantes diversas camadas da população e por democratizar atitudes capazes de beneficiar as práticas de controle.

Nesse contexto, objetivou-se avaliar o nível de conhecimento sobre a leishmaniose visceral e algumas atitudes preventivas em relação à doença em Belo Horizonte.

\section{Material e método}

Foi realizado um estudo longitudinal, caso-controle retrospectivo, no Município de Belo Horizonte, que está localizado na zona metalúrgica de Minas Gerais. Está situado entre os meridianos $43^{\circ} \mathrm{e} 45^{\circ}$, possui uma área de $335,5 \mathrm{~km}^{2}$, altitude de $858 \mathrm{~m}$ e clima predominantemente tropical. A temperatura média anual fica em torno de $20,5^{\circ} \mathrm{C}$ e há pequena variação de estações. $\mathrm{O}$ índice de precipitação pluvial é relativamente alto $(200 \mathrm{~mm}$ anuais), com a concentração de chuvas no período de novembro a março - 80\%-85\% (Instituto Nacional de Meteorologia, http://www.inmet. gov.br, acessado em 21/Out/2006).

Belo Horizonte foi selecionada devido à alta soroprevalência canina em vários anos consecutivos, à rápida evolução da doença e à presença constante e alarmante de casos e óbitos por leishmaniose visceral. $\mathrm{O}$ trabalho contou com o apoio/colaboração do município, por meio de recursos humanos e apoio logístico, além da disponibilidade de dados do Sistema de Informação de Agravos de Notificação (SINAN) por parte da Secretaria Municipal de Saúde, Prefeitura de Belo Horizonte.

Belo Horizonte possui uma população de 2.420.000 pessoas, distribuída em 612.838 imóveis 11,12 , e uma população canina estimada de 270.677 cães ${ }^{13}$, o que demonstra uma relação de 0,44 cão por imóvel e uma razão de 1 cão para 8,24 habitantes.

Belo Horizonte é dividida em nove administrações regionais com autonomia financeira e gerencial segundo a Prefeitura, e, de acordo com esta divisão, este estudo englobou todas as regionais.

O trabalho foi dividido em duas etapas: levantamento dos dados de campo e análise dos dados. As duas fases foram executadas entre os meses de janeiro a setembro de 2006 .
A obtenção de dados compreendeu um levantamento epidemiológico que se baseou nos casos de leishmaniose visceral humana ocorridos em Belo Horizonte, no ano de 2004. A amostra foi estimada com mínimo equivalente a 76 casos para que se obtivesse $95 \%$ de intervalo de confiança (IC95\%). Considerando-se a possibilidade de perdas de dados em situações de conhecimento insuficiente por parte do entrevistado, planejou-se uma amostra de 80 casos e 160 controles, totalizando assim 240 domicílios.

As visitas domiciliares foram compostas por leitura e assinatura do Termo de Consentimento Livre e Esclarecido e uso de questionário fechado e pré-codificado para cada grupo (caso ou controle) abrangendo informações individuais, questões relativas ao peri e intradomicílio, ao(s) cão(es) quando presente(s) e registro de imagens do peri e intradomicílio.

O estudo foi realizado em todos os domicílios em que houve concordância por escrito do morador, sendo este maior de 18 anos. Em situações em que ocorreram recusa ou domicílio fechado, houve substituição do caso a ser pesquisado por outro da lista do SINAN. Em se tratando dos domicílios-controles, a substituição foi pela unidade domiciliar seguinte.

As informações coletadas por meio das visitas domiciliares e do banco do SINAN, disponibilizado pela Prefeitura, foram codificadas e armazenadas utilizando-se o programa Stata versão 8.0 (Stata Corp., College Station, Estados Unidos).

As análises estatísticas utilizadas para ajuste de um modelo final logístico foram a descritiva, a univariada cujo ponto de corte foi de $p=0,20$ e a multivariada, no nível de significância de $0,0514,15$.

O estudo foi elaborado e executado segundo as diretrizes e normas que regem as pesquisas envolvendo seres humanos (Resolução $n^{o}$. 196/1996 do Conselho Nacional de Saúde) e aprovado pelos Comitês de Ética em Pesquisa da Universidade Federal de Minas Gerais (UFMG) e da Prefeitura de Belo Horizonte.

\section{Resultados e discussão}

Foram visitados 246 domicílios (82 casos e 164 controles) distribuídos geograficamente nas nove regionais administrativas de Belo Horizonte. A população caracteriza-se por ser adulta, com uma idade média de 36,7 anos, e mediana de 34 anos com IC95\%, havendo discreto predomínio do sexo feminino $(56,25 \%)$.

A distribuição da leishmaniose visceral foi notável em escolares de idade inferior ou igual a 10 anos (40,2\%). Estimou-se que para crianças 
menores de 10 anos o risco de contrair leishmaniose visceral aumenta em 109,77 vezes ao serem comparadas com indivíduos acima desta idade (Tabela 1). A susceptibilidade dessa faixa etária se deve, possivelmente, ao contato mais freqüente das crianças com animais, em comparação com adultos; além disso, os escolares apresentam as maiores taxas de carência nutricional e têm seu estado imunológico ainda em formação.

Marzochi et al. 16 e Nascimento et al. 17, encontraram um aumento do risco de contrair leishmaniose visceral com o avançar da idade, sugerindo um comportamento diferenciado da doença em grandes cidades como São Paulo, Brasil. Mas como verificado no presente estudo, o comportamento epidemiológico da leishmaniose visceral em Belo Horizonte ainda perfaz os índices costumeiros, não sendo ignorada a transição epidemiológica da doença que parece ocorrer no município, já que se tem percebido uma maior participação de adultos entre os acometidos. Em cidades de médio e pequeno portes, o perfil da leishmaniose visceral permanece praticamente inalterado; Badaró et al. 18 ao caracterizarem a epidemia em Feira de Santana (Bahia, Brasil) e Sherlock \& Almeida 19, no foco de Jacobina (Bahia), verificaram que os maiores índices de prevalência e incidência da doença ainda recaem sobre as crianças. Quanto aos sintomas, Araújo 20 verificou que estes se manifestam mais severamente nas crianças.

Em relação ao sexo, a leishmaniose visceral demonstrou ser mais freqüente em homens (62,2\%), sendo estimado que eles têm 2,57 vezes mais chances de contrair leishmaniose visceral que a mulher (Tabela 2). Essa disparidade entre gêneros ainda permanece sem explicação científica, mas provavelmente a maior exposição masculina acontece pelo mais freqüente trânsito do trabalho para casa em horários que coincidem com os de alimentação do flebótomo. Esse fato já fora assinalado por Alencar 21 e Marzochi et al. 22 .

Ao realizarem um estudo de coorte na Etiópia, Ali \& Ashford 23 observaram que ambos os sexos foram infectados, porém os homens tiveram duas vezes mais chances de desenvolver a doença que as mulheres; tanto as taxas de soroconversão quanto a conversão de testes cutâneos foram maiores em homens que em mulheres, principalmente em idosos, por terem o sistema imunológico menos resistente. Deane \& Deane 24 e Nascimento et al. 17 não descartam a possibilidade da susceptibilidade quanto a leishmaniose visceral ter fundamento genético.

Quanto à escolaridade, os entrevistados detinham baixo índice de instrução, já que 68,3\% podiam ser incluídos no grupo daqueles que não
Tabela 1

Risco de adoecer de leishmaniose visceral segundo a variável idade, selecionada na análise univariada, de acordo com o nível de significância de 0,20. Belo Horizonte, Minas Gerais, Brasil, 2006.

\begin{tabular}{lccc}
\hline Variável & OR & Valor de $\mathbf{p}$ & IC95\% \\
\hline Idade (anos) & 0,97 & 0,000 & $0,95-0,97$ \\
$<6$ & 56,11 & 0,000 & $7,39-426,21$ \\
$<10$ & 109,77 & 0,000 & $14,64-823,30$ \\
\hline
\end{tabular}

Tabela 2

Distribuição da variável sexo, selecionada na análise univariada, segundo o nível de significância de 0,20. Belo Horizonte, Minas Gerais, Brasil, 2006.

\begin{tabular}{lcccccc}
\hline Variável & \multicolumn{2}{c}{ Caso $(\mathbf{N}=\mathbf{8 2})$} & \multicolumn{2}{c}{ Controle $(\mathbf{N}=164)$} & OR & p \\
& $\mathbf{n}$ & $\%$ & $\mathbf{n}$ & $\%$ & \\
\hline $\begin{array}{l}\text { Sexo } \\
\text { Feminino }\end{array}$ & 31 & 37,8 & 100 & 61,0 & - & - \\
Masculino & 51 & 62,2 & 64 & 39,0 & - & - \\
\hline
\end{tabular}

chegaram a terminar o ensino médio, aqui denominado ainda como segundo grau devido à maior democratização deste termo perante os entrevistados. Observou-se ainda que $41,5 \%$ dos casos possuíam apenas o primário, $26,8 \%$ nunca haviam freqüentado uma escola e $2,4 \%$ possuíam nível superior completo.

Nos controles vê-se uma diminuição significativa de analfabetos (4,3\%) e uma distribuição mais descentralizada da escolaridade, pois $22 \%$ têm primeiro grau incompleto e $17,1 \%$ o segundo grau completo.

A variável escolaridade demonstrou ainda que uma pessoa que nunca freqüentou uma escola, ou que se classifica como analfabeto, tem oito vezes mais chances de ser acometido por leishmaniose visceral do que um indivíduo alfabetizado (Tabela 3). Essa associação pode ser explicada pela maioria dos analfabetos estarem entre a população de baixo poder aquisitivo, que também é a que mais sofre com a doença, segundo Bevilacqua et al. 4. A educação em saúde, praticada atualmente em diversos momentos durante a formação estudantil, tem forte potencial de controle epidemiológico, com isto, a limitação gerada pela ausência ou baixa escolaridade parece se refletir na também ausente ou minimizada prática preventiva. 
Distribuição da escolaridade e seus resultados da análise univariada, segundo o nível de significância de 0,20. Belo Horizonte, Minas Gerais, Brasil, 2006.

\begin{tabular}{|c|c|c|c|c|c|c|}
\hline \multirow[t]{2}{*}{ Variável } & \multicolumn{2}{|c|}{ Caso $(\mathrm{N}=82)$} & \multicolumn{2}{|c|}{ Controle $(N=164)$} & \multirow[t]{2}{*}{ OR } & \multirow[t]{2}{*}{$p$} \\
\hline & $\mathbf{n}$ & $\%$ & $n$ & $\%$ & & \\
\hline Escolaridade & & & & & - & - \\
\hline Nunca freqüentou a escola & 22 & 26,8 & 7 & 4,3 & 8,22 & 0,000 \\
\hline Primário & 34 & 41,5 & 34 & 20,7 & 0,31 & 0,021 \\
\hline 1ㅇ grau incompleto & 4 & 4,9 & 36 & 22,0 & 0,03 & 0,000 \\
\hline 1ㅇ grau completo & 3 & 3,7 & 26 & 15,9 & 0,03 & 0,000 \\
\hline 2o grau incompleto & 8 & 9,8 & 23 & 14,0 & 0,11 & 0,000 \\
\hline 2o grau completo & 7 & 8,5 & 28 & 17,1 & 0,07 & 0,000 \\
\hline 3o grau incompleto & 2 & 2,4 & 5 & 3,0 & 0,12 & 0,029 \\
\hline 3o grau completo & 2 & 2,4 & 5 & 3,0 & 0,12 & 0,029 \\
\hline
\end{tabular}

Em relação ao conhecimento sobre a leishmaniose visceral, verificou-se que $50 \%$ dos indivíduos acometidos pela doença desconheciamna completamente quando foram infectados, $26,8 \%$ já tinham ouvido falar da doença, 3,7\% conheciam os sintomas e apenas 1,2\% conhecia o vetor. Entre os controles, a leishmaniose visceral era desconhecida para $30,5 \%$ do grupo, $40,2 \%$ já tinham ouvido falar da doença, $4,3 \%$ conheciam os sintomas e apenas $3 \%$ conheciam o vetor (Tabela 4). A precariedade de informação sobre a leishmaniose visceral, traduzida nesses resultados, aponta a necessidade da realização de práticas educativas em diferentes frentes, que podem contar com a participação de médicos e veterinários, durante consultas, professores e agentes de saúde em palestras e durante as visitas domiciliares.

O quase desconhecimento do vetor e dos sintomas pela população entrevistada, está de acordo com as observações feitas por Luz et al. $10 \mathrm{em}$ Belo Horizonte. Os autores, durante avaliação do potencial dos folhetos sobre a leishmaniose visceral como instrumentos de informação, tanto para profissionais da área de saúde como para leigos, também encontraram maior desconhecimento e confundimento no que se refere aos sintomas da leishmaniose visceral. Já em estudo feito por Gama et al. 25, no Maranhão, Brasil, o desconhecimento sobre a prevenção da doença obteve o maior percentual $(77,8 \%$ dos entrevistados não sabiam como prevenir a leishmaniose visceral)

A variável conhecer nome do vetor mostrou que pessoas que dizem conhecer, ao menos um nome dado ao vetor, tem 1,58 vez mais chance de serem acometidas pela leishmaniose visceral que aquelas que desconhecem os nomes (Tabela 5). Essa associação pode ser explicada pelo fato desse conhecimento existir devido a um contato prévio com a doença, seja por pessoas ou animais próximos infectados. Em General Carneiro (Minas Gerais), Moreno et al. 28 apesar de terem encontrado uma odds ratio (OR) de 0,4 para a variável conhecer cão com leishmaniose, não desmereceu o potencial de risco do conhecimento prévio da doença, sendo o resultado atribuído ao conhecimento similar de ambos os grupos comparados.

Qualquer conhecimento sobre a leishmaniose visceral (exceto saber nomes do vetor como foi dito anteriormente) foi considerado fator de proteção, capaz de minimizar o risco de ocorrência de leishmaniose visceral em 2,24 vezes. Tal dado também foi encontrado por Dias $9 \mathrm{e}$ Cabrera 27.

Diante das medidas de controle, passíveis de serem praticadas pelos moradores, observou-se que $56,4 \%$ dos casos não praticam nenhum tipo de prevenção referente à doença, 2,5\% possuem cães, porém não os levam ao veterinário, e 4,9\% possuem cães e levam os animais ao veterinário.

No grupo controle, $40,2 \%$ dos entrevistados reconheceram não tomar nenhuma medida de prevenção quanto à leishmaniose visceral, 8,5\% têm cães, mas não os levam ao veterinário, e 7,9\% possuem cães e levam os animais ao veterinário.

Foi possível estimar diminuição de 1,94 vez no risco de ocorrência de leishmaniose visceral para pessoas que declararam tomar qualquer atitude preventiva, quando comparadas com pessoas que não praticam tais ações (Tabela 6). Em se tratando de evitar animais, inclusive cães, como forma de prevenção da doença, nos con- 
Distribuição das variáveis categóricas relacionadas ao conhecimento dos moradores sobre a leishmaniose visceral, e seus respectivos resultados na análise univariada, segundo o nível de significância de 0,20. Belo Horizonte, Minas Gerais, Brasil, 2006.

\begin{tabular}{|c|c|c|c|c|c|c|}
\hline \multirow[t]{2}{*}{ Variável } & \multicolumn{2}{|c|}{ Caso $(\mathrm{N}=82)$} & \multicolumn{2}{|c|}{ Controle $(\mathrm{N}=164)$} & \multirow[t]{2}{*}{ OR } & \multirow[t]{2}{*}{$\mathrm{p}$} \\
\hline & $\mathrm{n}$ & $\%$ & $\mathrm{n}$ & $\%$ & & \\
\hline \multicolumn{7}{|l|}{ Conhecimento sobre a } \\
\hline \multicolumn{7}{|l|}{ leishmaniose visceral } \\
\hline Nenhum conhecimento & 41 & 50,0 & 50 & 30,5 & 0,44 & 0,004 \\
\hline Já ouviu falar & 22 & 26,8 & 66 & 40,2 & 0,40 & 0,006 \\
\hline Sabe da relação com o cão & 4 & 4,9 & 13 & 7,9 & 0,37 & 0,110 \\
\hline Sabe do vetor & 1 & 1,2 & 5 & 3,0 & 0,24 & 0,208 \\
\hline Sabe alguns sintomas & 3 & 3,7 & 7 & 4,3 & 0,52 & 0,372 \\
\hline Sabe razoavelmente da doença & 11 & 13,4 & 23 & 14,0 & 0,58 & 0,207 \\
\hline
\end{tabular}

Tabela 5

Distribuição das variáveis dicotômicas relacionadas ao conhecimento da população perante a leishmaniose visceral e seus respectivos resultados na análise univariada, segundo o nível de significância de 0,20. Belo Horizonte, Minas Gerais, Brasil 2006.

\begin{tabular}{llll}
\hline Variável & OR & p > Izl & IC95\% \\
\hline Conhece nomes do vetor & 1,58 & 0,121 & $0,88-2,85$ \\
Conhece forma de transmissão & 1,18 & 0,536 & $0,06-2,05$ \\
\hline
\end{tabular}

Tabela 6

Distribuição das atividades preventivas e seus respectivos resultados na análise univariada, segundo o critério de significância no nível de 0,20. Belo Horizonte, Minas Gerais, Brasil, 2006.

\begin{tabular}{|c|c|c|c|c|c|c|}
\hline \multirow[t]{2}{*}{ Variável } & \multicolumn{2}{|c|}{ Caso $(\mathbf{N}=82)$} & \multicolumn{2}{|c|}{ Controle $(\mathrm{N}=164)$} & \multirow[t]{2}{*}{ OR } & \multirow[t]{2}{*}{$p$} \\
\hline & n & $\%$ & $\mathbf{n}$ & $\%$ & & \\
\hline \multicolumn{7}{|l|}{ Atitudes preventivas praticadas } \\
\hline pelos entrevistados & & & & & & 1,940 \\
\hline Não pratica nenhuma & 47 & 56,8 & 66 & 40,2 & 0,51 & 0,015 \\
\hline \multicolumn{7}{|l|}{ Tem cães, mas não leva ao } \\
\hline veterinário & 2 & 2,5 & 14 & 8,5 & 0,20 & 0,040 \\
\hline Tem cães, mas leva ao veterinário & 4 & 4,9 & 13 & 7,9 & 0,43 & 0,167 \\
\hline Não tem cães & 2 & 2,5 & 11 & 6,7 & 0,25 & 0,086 \\
\hline Evita criar animais, inclusive o cão & 6 & 7,4 & 22 & 13,4 & 0,38 & 0,056 \\
\hline Melhorou a limpeza da casa & 18 & 22,2 & 34 & 20,7 & 0,74 & 0,406 \\
\hline Outras & 3 & 3,7 & 4 & 2,4 & 1,05 & 0,941 \\
\hline
\end{tabular}


troles o percentual foi superior ao encontrado nos casos (13,4\% e 7,4\%, respectivamente).

A limpeza do domicílio surge como a segunda forma de prevenção mais citada $(22,2 \%$ para casos e $20,7 \%$ para controles). Porém, deve ser destacada que a limpeza do domicílio em boa parte das entrevistas não foi citada de forma específica para leishmaniose visceral, e sim como uma prática do cotidiano dos moradores. Dessa forma, essa variável deve ser avaliada com certo critério. Como foi dada a liberdade aos entrevistados para citarem outras formas de prevenção, em ambos os casos foi relatado o uso de citronela (Cymbopogon flexuosus) e de outros repelentes, durante os horários de alimentação dos vetores.

A prática de ações preventivas perante doenças, como a leishmaniose visceral, ainda necessita de melhor divulgação e de incentivo por parte do governo e da própria sociedade. Mudanças de atitudes numa população é meta a ser atingida com o tempo, pois envolve variações na cultura; esta parece ser a explicação para a dificuldade de se alcançar altos índices de prevenção.

A análise de regressão logística realizada no presente estudo obteve um modelo final composto por cinco variáveis significativas (idade, sexo, escola, conhecimento sobre a leishmaniose visceral e desconhecimento total do entrevistador sobre a leishmaniose visceral), no nível de significância de 0,05 . Esse modelo teve poder de explicação equivalente a $70,7 \%$. Com isso, tornou-se possível analisar o peso das variáveis de forma conjunta, numa tentativa de recriar a realidade (Tabela 7).

Ao final das análises, é atribuído à variável idade uma diminuição de 0,96 vez no risco de ocorrência da leishmaniose visceral (Tabela 7).
O fato da pessoa ser do sexo masculino aumenta seu risco de adoecer em 3,7 vezes, quando comparada à outra do sexo feminino. A escolaridade é fator de proteção que diminui o risco de ter leishmaniose visceral em 0,64 vez.

Saber algo sobre a leishmaniose visceral, seja em relação às formas de transmissão ou a características do vetor, diminui o risco em 0,79 vez. No entanto, não conhecer a doença se mostra como fator de proteção no presente trabalho, diminuindo o risco de ter leishmaniose visceral em 2,57 vezes. Tal fato se deve à ausência de contato prévio com a doença.

\section{Conclusão}

Em Belo Horizonte, o nível de conhecimento da população em relação à leishmaniose visceral se restringe a informações superficiais sobre a doença e a atitudes preventivas inespecíficas, o que dificulta a implementação eficiente de práticas de controle da leishmaniose visceral.

A realização deste estudo em uma capital brasileira, que sofre o avanço da leishmaniose visceral, contribui para a melhor compreensão da epidemiologia desta doença em áreas urbanas e salienta a necessidade de se manter as medidas de controle já realizadas com um diferencial, o da educação em saúde.

O potencial de proteção que tem o conhecimento sobre a leishmaniose visceral deixa claro que ao se tornar consciente do agravo a população tem como contribuir, de forma ativa e permanente, no controle do mesmo, sendo esta a chave para a execução, consolidação e vigilância das ações de controle das endemias como a leishmaniose visceral.

Tabela 7

Modelo final de regressão logística no nível de significância de 0,05.

\begin{tabular}{lccc}
\hline Variável & OR & p > |z| & IC95\% \\
\hline Idade & 0,96 & 0,000 & $0,94-0,97$ \\
Sexo & 3,71 & 0,000 & $1,92-7,16$ \\
Escola & 0,64 & 0,000 & $0,53-0,77$ \\
Conhece a leishmaniose visceral & 0,79 & 0,036 & $0,64-0,98$ \\
Não conhece a leishmaniose visceral & 2,57 & 0,023 & $1,13-5,80$
\end{tabular}




\section{Resumo}

Objetivou-se avaliar o nível de conhecimento e algumas atitudes preventivas em relação à leishmaniose visceral em Belo Horizonte, Minas Gerais, Brasil, em 2006. Foi feito um estudo de caso-controle, com visitas domiciliares e questionário semi-estrurado. Comparou-se dois grupos: (1) 82 casos humanos de leishmaniose visceral ocorridos em 2004 e (2) 164 controles, constituídos por vizinhos dos casos. A leishmaniose visceral acometeu mais em crianças, com aumento do risco de contrair leishmaniose visceral de 109,77 vezes para menores de dez anos. O homem demonstrou ter 2,57 vezes mais chances de adoecer que a mulher. A escolaridade da população mostrou-se baixa (68,3\% não completaram o ensino médio). Cinqüenta por cento dos casos desconheciam-na quando foram infectados e apenas 1,2\% conhecia o vetor. Conhecer algo sobre a leishmaniose visceral minimizou o risco de adoecer em 2,24 vezes. Quanto às atitudes de proteção, o risco de se contrair leishmaniose visceral diminui em 1,94 vez para pessoas que mantêm limpos os domicílios ou que levam o cão ao veterinário. Em Belo Horizonte, o conhecimento da população perante a leishmaniose visceral é superficial e as atitudes preventivas inespecíficas.

Leishmaniose Visceral; Fatores de Risco; Conhecimento; Prevenção de Doenças

\section{Colaboradores}

B. K. A. Borges dedicou-se a execução do trabalho de campo e levantamento bibliográfico para o trabalho e elaborou juntamente com J. A. Silva o referencial teórico, metodologia, resultados, discussão e adequação às normas dos Cadernos de Saúde Pública. J. P. A. Haddad foi responsável pela parte estatística do artigo. D. F. Magalhães e L. M. L. Ribeiro participaram da coleta do material durante a pesquisa de campo. E. C. Moreira eV. O. P. Fiúza participaram da discussão e das etapas de elaboração e execução do projeto de pesquisa. Todos os colaboradores participaram da revisão final do texto.

\section{Referências}

1. Barata RA, Silva JCF, Silva JC, Prata A, Macedo CG, Fiuza JA, et al. Aspectos da ecologia e do comportamento de flebotomíneos em área endêmica de leishmaniose visceral. Rev Soc Bras Med Trop 2005; 39:421-5.

2. Alencar JE. Expansão do calazar no Brasil. Ceará Méd 1983; 5:86-102.

3. Secretaria de Vigilância em Saúde, Ministério da Saúde. Manual de vigilância e controle da leishmaniose visceral. Brasília: Secretaria de Vigilância em Saúde, Ministério da Saúde; 2006.

4. Bevilacqua PD, Paixão HH, Modena CM, Castro MCPS. Urbanização da leishmaniose visceral em Belo Horizonte. Arq Bras Med Vet Zootec 2001; 53:1-8.

5. Carandina L, Magaldi C. Análise das condições de saúde e de vida da população urbana de Botucatu, SP (Brasil). II - Conhecimentos e opiniões da população sobre sintomas de doenças, 1983. Rev Saúde Pública 1989; 23:196-206.
6. Silva ES, Gontijo CMF, Pacheco RS, Fiúza VOP, Brazil RP. Visceral leishmaniasis in Metropolitan Region of Belo Horizonte, State of Minas Gerais, Brazil. Mem Inst Oswaldo Cruz 2001; 96:285-91.

7. Fundação Nacional de Saúde. Leishmaniose visceral no Brasil. Boletim Eletrônico Epidemiológico. http://www.funasa.gov.br/Web520funasa/ pub/boletinr/pdf/informe.pdf (acessado em 10/ Mai/2005).

8. Secretaria Municipal de Saúde, Prefeitura de Belo Horizonte. Dados demográficos. http:// portall.pbh.gov.br/pbh/index.html?id conteudo=2757\&id_nivell=-1 (acessado em 12/ Nov/2005).

9. Dias JCP. Problemas e possibilidades de participação comunitária no controle das grandes endemias do Brasil. Cad Saúde Pública 1998; 14 Suppl 2:S19-37. 
10. Luz ZMP, Schall V, Rabello A. Evaluation of a pamphlet on visceral leishmaniasis as a tool for providing disease information to healthcare professionals and laypersons. Cad Saúde Pública 2005; 21:608-21.

11. Instituto Brasileiro de Geografia e Estatística. Censo demográfico 2000. http:/ / www.ibge.gov.br/ censo2000 (acessado em 24/Abr/2005).

12. Prefeitura de Belo Horizonte. Imposto predial e territorial urbano, 2004. http://www.fazenda.pbh. gov.br/iptu_guias/iptu00.asp (acessado em 05/ Jun/2005).

13. Secretaria de Vigilância do Centro de Zoonoses, Secretaria Municipal de Saúde. Censo canino 2000. http://www.pbh.gov.br (acessado em 06/ Mai/2005).

14. Schlesselman JJ. Case-control studies: design, conduct, analysis. New York: Oxford University Press; 1982.

15. Medronho RA, Carvalho DM, Bloch KV, Luiz RR, Werneck GL. Epidemiologia. São Paulo: Editora Atheneu; 2004.

16. Marzochi MCA, Sabroza PC, Toledo LM, Marzochi KBF, Tramontano NC, Rangel Filho FB. Leishmaniose visceral na cidade do Rio de Janeiro - Brasil. Cad Saúde Pública 1985; 1:5-17.

17. Nascimento MDSB, Costa JML, Fiori BIP, Viana JMC, Filho MSG, Alvim AC, et al. Aspectos epidemiológicos determinantes na manutenção da leishmaniose visceral no Estado do MaranhãoBrasil. Rev Soc Bras Med Trop 1996; 29:233-40.

18. Badaró R, Jones TC, Lorenzo R, Cerf J, Sampaio D, Carvalho EM, et al. A prospective study of visceral leishmaniasis in an endemic area of Brazil. J Infect Dis 1986; 154:639-49.

19. Sherlock IA, Almeida SP. Observações sobre o calazar em Jacobina. Bahia. II - leishmaniose canina Rev Bras Malariol Doenças Trop 1969; 21:535-9.
20. Araújo TM. Caracterização da epidemia e avaliação das medidas de controle no Município de Feira de Santana, de 1995 a 2000 [Tese de Doutorado]. Salvador: Universidade Federal da Bahia; 2001.

21. Alencar JE. Calazar canino: Contribuição para o estudo da epidemiologia do calazar no Brasil [Tese de Doutorado]. Fortaleza: Universidade Federal do Ceará; 1956.

22. Marzochi MCA, Marzochi KBF, Carvalho RW. Visceral leishmaniasis in Rio de Janeiro. Parasitol Today 1994; 10:34-7.

23. Ali A, Ashford RW. Visceral leishmaniasis in Etiópia. III. The magnitude and annual incidence of infection, as measured by serology in an endemic area. Ann Trop Med Parasitol 1994; 88:43-7.

24. Deane LM, Deane MP. Visceral leishmaniasis in Brazil: geographical distribution and transmission. Rev Inst Med Trop São Paulo 1962; 4:198-212.

25. Gama MEA, Barbosa JS, Pires B, Cunha AKB, Freitas AR, Ribeiro IR, et al. Avaliação do nível de conhecimento que populações residentes em áreas endêmicas têm sobre leishmaniose visceral, Estado do Maranhão, Brasil. Cad Saúde Pública 1998; 14:381-90.

26. Moreno E, Melo MN, Antunes CMF, Lambertucci JR, Seruto JC, Andrade-Ribeiro AS, et al. Epidemiologia da leishmaniose visceral humana assintomática em área urbana, Sabará, Minas Gerais, 19981999. Inf Epidemiol SUS 2002; 11:37-9.

27. Cabrera GP, Silva VO, Costa RT, Reis AB, MayrinkW, Genaro O, et al. The fucose-mannose ligant-ELISA in the diagnosis and prognoses of canine visceral leishmanasis in Brazil. Am J Trop Med Hyg 1999; 61:296-301.

Recebido em 01/Fev/2007

Versão final reapresentada em 30/Abr/2007

Aprovado em 16/Mai/2007 